\title{
Cardiac and Vascular Responses to Thigh Cuffs and Respiratory Maneuvers on Crewmembers of the International Space Station
}

Douglas Hamilton, M.D., Ph.D. ${ }^{1}$, Ashot E. Sargsyan, M.D ${ }^{1}$., Kathleen Garcia, R.D.C.S. ${ }^{1}$ Douglas Ebert, Ph.D. ${ }^{1}$, Peggy A. Whitson, Ph.D. ${ }^{2}$, Alan Feiveson, PhD. ${ }^{2}$, Irina V. Alferova, MD, Ph.D. ${ }^{3}$, Scott A. Dulchavsky, M.D., Ph.D. ${ }^{4}$, Vladimir P. Matveev ${ }^{5}$, M.D., Valery V. Bogomolov $^{5}$, M.D., Ph.D., J. Michael Duncan, M.D. ${ }^{2}$

${ }^{1}$ Wyle Integrated Science and Engineering, Houston, TX

${ }^{2}$ NASA Lyndon B. Johnson Space Center (JSC), Houston, TX, USA

${ }^{3}$ Institute of Biomedical Problems (IBMP) of the Russian Academy of Sciences, Moscow, Russian Federation

${ }^{4}$ Henry Ford Hospital, Detroit, MI

${ }^{5}$ Yuri A. Gagarin Cosmonaut Training Center (GCTC), Star City, Russian Federation

Primary funding for this project was provided by the Exploration Medical Capability (ExMC) element of NASA's Human Research Program (HRP), through the Bioastronautics Contract to Wyle Integrated Science and Engineering Group (NAS9-02078). 
Key Words

Adaptation, Physiological: *physiology

Adult

Astronaut

Blood Volume: physiology

Cardiac Function

Cardiac mass

Cardiac Output: physiology

Echocardiography

Female

Heart: physiology

Humans

Male

Microgravity

Stroke Volume: physiology

*Weightlessness

$\begin{array}{ll}\text { Abbreviations List } \\ \text { CVP } & \text { - Central Venous Pressure } \\ \text { ECHO } & \text { - Cardiac and Vascular Ultrasound } \\ \text { FDR } & \text { - False Discovery Rate } \\ \text { HDT } & \text { - Head Down Tilt } \\ \text { IJV } & \text { - Internal Jugular Vein } \\ \text { ISS } & \text { - International Space Station } \\ \text { JVP } & \text { - Jugular Venous Pressure } \\ \text { LA } & \text { - Left Atrial } \\ \text { LV } & \text { - Left Ventricular } \\ \text { MCC-H } & \text { - Mission Control Center - Houston } \\ \text { RA } & \text { - Right Atrial } \\ \text { RV } & \text { - Right Ventricular } \\ \text { TD } & \text { - Tissue Doppler } \\ \text { US } & \text { - Ultrasound }\end{array}$

Word Count Abstract $=240$

Word Count Manuscript $=4703$

References $=43$ 


\section{Abstract}

Background: The transition to microgravity eliminates the hydrostatic gradients in the vascular system. The resulting fluid redistribution commonly manifests as facial edema, engorgement of the external neck veins, and a decrease in leg diameter. This experiment examined the responses to modified Valsalva and Mueller maneuvers measured by cardiac and vascular ultrasound (ECHO) in a baseline steady state and during preload reduction introduced with thigh occlusion cuffs used as a counter-measure device (Braslet cuffs) measured by cardiac and vascular ultrasound examinations.

Methods: Nine International Space Station crewmember subjects (Expeditions 16 - 20) were examined in 15 experiment sessions 101 \pm 46 .days after launch (mean \pm SD; 33 - 185). Twenty Seven cardiac and vascular parameters were obtained with/without respiratory maneuvers before and after tightening of the Braslet cuffs.

Results: Non-physicians performed diagnostic-quality cardiac and vascular ultrasound examinations using remote guidance. Three of 27 combinations of maneuvers and Braslet or Braslet alone were identified as being significant changed when compared to baseline. Eleven of 81 differences between combinations of Mueller, Valsalva or baseline were significant and related to cardiac preload reduction or increase in lower extremity venous volume.

Conclusions: Acute application of Braslet occlusion cuffs causes lower extremity fluid sequestration and exerts commensurate measurable effects on cardiac performance in microgravity. Ultrasound techniques to measure the hemodynamic effects of thigh cuffs in combination with respiratory maneuvers may serve as an invaluable tool in determining the volume status of the cardiac patient at the 'microgravity bedside'. 


\section{Introduction}

An immediate effect of the transition to microgravity during early spaceflight is a loss of the hydrostatic gradient in the venous vasculature, resulting in a cephalad fluid shift. $(1 ; 2)$ The estimated loss of lower extremity volume is in the order of 1to 2 liters, (1;3) which is larger than the shifts seen on Earth when moving from upright to supine or even head-down-tilt posture. While the mechanisms that mitigate the pooling of blood in the lower extremities apparently continue to act in the absence of gravity, central venous pressure (CVP) has been measured invasively during spaceflight and noted to decrease by about 5- to 7- $\mathrm{mmHg}$, with no clinically significant long-term changes in cardiac output.(4;5) The bedside assessment of the jugular venous pressure (JVP) on Earth is a reliable indicator of right atrial pressure because the vena cava acts as a venous hydrostatic column of blood. Jugular venous distension under microgravity conditions is seen normally and persists(6) throughout the mission, reducing its effectiveness as an index of right atrial pressure and right ventricular preload.

The effect of fluid redistribution in astronauts is commonly observed as visible facial edema ("puffiness") and engorgement of the external neck veins, with a concomitant decrease in leg size. Subjectively, crewmembers report "fullness in the head," nasal congestion, and diminished sense of taste and smell.(7) Previous investigations have documented the peripheral vascular effects of the Braslet-M occlusion thigh cuff and have suggested that it also has an effect on cardiac preload, however ultrasound technology at the time was not adequate to confirm this.(6) The International Space Station (ISS) Ultrasound System operated with realtime remote guidance has heightened the capability of acquiring clinically and operationally valuable ultrasound imaging data from orbit. $(6 ; 8-12)$

The Braslet-M thigh cuff countermeasure device is intended to ameliorate the symptoms associated with the microgravity-induced cephalad volume shifts in the early hours and days of microgravity exposure through obstruction of venous outflow and commensurate fluid sequestration in the lower extremities. The cuffs are custom built for each crewmember before the mission and consist of segments of elastic and non-elastic materials to conform to the shape of the upper thigh. (Figure 1) Each device contains an adjustment belt which can be tightened to achieve the desired degree of compression based on preflight calibration data. The Braslet-M 
device also has been used in space to "retrain" the cardiovascular system in the final days of long-duration missions and prevent orthostatic hypotension upon landing.

The custom-made cuffs are calibrated in a special preflight tilt-table procedure, and then flown as personal equipment on the ISS. During the calibration procedure, trained experts use subjective responses and cranial rheographic data to determine the setting of the device, which is expected to provide appropriate compression of the extremity during the space flight.

Research projects involving echocardiography have been conducted on almost every space vehicle since 1980.(6;13-21) In a recent ISS-based study, Hamilton et al(6) presented a set of standardized methods and echocardiographic "normal" results in six subjects during long duration spaceflight. The current investigation is a collaborative effort between the NASA and the Federal Space Agency of Russia/Roscosmos investigators to observe the hemodynamic effects of the Braslet-M device in nominal steady-state condition, as well as during Mueller and Valsalva respiratory maneuvers performed before and during its application. We believe Braslet manipulates central venous pressure and will measurably alter a number of circulatory variables.(22) Echocardiographic and vascular parameters were acquired during the sudden release of the occlusion device.

\section{Hypothesis}

The Braslet-M device, when used per the current calibration and procedures, will:

- consistently cause fluid sequestration and reduce flow velocities in the venous vascular bed of lower extremities in microgravity, acutely reducing the circulating volume;

- reduce cardiac preload in microgravity for the duration of application;

- facilitate the effects of the Valsalva and Mueller maneuvers on the filling (distention) and behavior of internal jugular vein observed by real-time sonography.

\section{Methods}

\section{Subject Selection}

All ISS crewmembers were medically certified for space flight by the ISS Multilateral 
Space Medicine Board using a comprehensive physical examination and screening that includes a comprehensive cardiovascular assessment with ECG, exercise stress test, transthoracic echocardiography, Holter monitoring, Electron Beam CT for coronary calcium scoring, a large assortment of laboratory blood tests, and a clinical cardiovascular risk assessment. Subjects and operators for this study were enlisted on a voluntary basis following informed consent. The study was approved by the human research boards of each subject's agency, and then by the ISS Human Research Multilateral Review Board.

\section{Braslet Calibration}

Braslet-M sets were custom made by the manufacturer (Kentavr-Nauka, Ltd., Moscow, Russia) for each subject and then individually calibrated as a nominal operational countermeasure in a standardized preflight tilt-table procedure. Cranial rheographic data were acquired in the baseline state (supine) and then in $-30^{\circ}$ head-down position. The Braslet was gradually tightened in a step-wise fashion while trained experts recorded subjective responses and followed rheographic tracing and the appearance of the subject. The setting of the device was thus determined to assure subjective improvement (reduction of fullness of the head, nasal congestion, and often a perception of not being tilted) as well as return of rheographic tracing to the baseline type. (Figure 1) This setting is considered to provide appropriate compression of the extremity for operational purposes, therefore the same setting was used in this study.

\section{Training}

Approximately four months before launch, each subject received two hours of operator and/or subject familiarization, including hands-on instruction by an expert sonographer using a simulated flight control setting. All training was performed in the U.S. Destiny module Human Research Facility simulator (Johnson Space Center - Houston) containing a flight-modified ATL HDI-5000 (ATL/Philips, Seattle, USA) ultrasound system. The P4-2 phased array cardiac ultrasound probe (1-4 MHz) was used for cardiac imaging, and L12-5 linear array probe was used to demonstrate the vascular imaging procedures. This configuration was identical to the actual set up in the Destiny module of the ISS.

\section{On Orbit Data Acquisition}


The study spanned three consecutive ISS Expeditions with six, five and four sessions respectively involving nine crewmembers from 33 to 185 days after launch $(101 \pm 46$, mean \pm SD). No ground data were collected before or after the mission, as each crewmember served as their own control.

During each on-orbit session, real-time video from the ISS ultrasound system was transmitted to Mission Control Center - Houston (MCC-H) using the ISS video subsystem. Two-way private audio was provided to the expert sonographer in MCC-H. The crew-members were remotely guided through the study protocol, which typically took 60 minutes to perform. (Figure 2.)

The astronauts were allowed to refine specific restraining techniques for both the subject and the operator to allow unrestricted use of the keyboard, access to the thoracic region and both thigh areas of the subject, and assure mutual stability for the examination in the microgravity environment. Attention was paid to the prevention of hand fatigue of the operator. In some cases, the subject assisted with ultrasound keyboard manipulations.

The cardiac ultrasound examination was conducted using the four-chamber apical view primarily, with sub-costal four-chamber view as the alternative, and involved B, M, Pulsed Wave Doppler, and Tissue Doppler modes. Probe repositioning and change-outs were minimized to enhance image acquisition and reduce examination duration. (Figure 3) The probe position was optimized per the remote sonographer's voice commands to achieve the desired images. The remote guidance team made real-time decisions on the quality of the apical view and the need for an alternative approach. All sonographers were registered diagnostic cardiac sonographers (RDCS) and registered vascular technologists (RVT) by the American Registry for Diagnostic Medical Sonography (ARDMS). (23)

All sessions were conducted in a consistent manner with cardiac and peripheral vascular ultrasound being acquired first without Braslet during three test conditions (baseline, a modified Valsalva maneuver, and a modified Mueller maneuver), and then under the same conditions after the Braslet had been on for 10 to 30 minutes. The modified respiratory maneuvers were performed by exhaling or inhaling through a plastic tube of standardized length and diameter to limit airway pressures to less than $\pm 40 \mathrm{~cm}_{2} \mathrm{O}$.(Figure 2) The subjects were trained to spread the respiratory effort over 8 to10 seconds starting at baseline, which was demonstrated in ground experiments to maintain the airway pressure within the above limits. At the end of the 'Braslet- 
On" session, a cardiac four chamber or RV TD was obtained during the rapid release of the Braslet to observe the effect of the fluid sequestered in the lower extremities returning to the effective circulating volume.

All the ultrasound images were reviewed and analyzed by registered sonographers and a cardiologist certified by the National Board of Echocardiography(24) external to the investigator group, to verify the diagnostic quality of the data. Depending on the quality of the data, all the parameters listed in Table 1 were measured in three different frames or cardiac cycles. In some subjects, data points from a particular step of the protocol were rejected due to poor quality; however, data of acceptable fidelity were obtained from all subjects.

\section{Statistical Methods}

Data analysis consisted of two phases. Phase I was an exploratory analysis designed to identify outcome parameters (Table 1) that showed evidence of being affected by the Braslet for any or all of the test conditions. For this phase, we made no distributional assumptions and used Somers $D$, a non-parametric measure of association to describe consistency of change with regard to Braslet status (on or off) taking into account differences between subjects and possibly flight days within a mission for the same subject. In Table 1, combinations of parameters and test conditions were flagged ( see 'stars' Table 1) as showing statistically significant change if Pvalues for the test $\mathrm{H}_{0}: D=0$ were below the critical threshold of 0.019 , as determined by controlling the false discovery rate to $10 \%$ using the method of Benjamini, Krieger and Yekutieli(25) with a nominal test level of $\alpha=.05$. The false discovery rate (FDR) is the expected proportion of rejected hypotheses that in fact were null and should not have been rejected.

In Phase II, we used a parametric mixed model to describe each parameter's response, with and without the Braslet, and under each of the three test conditions (baseline, Valsalva, Mueller). For some outcome parameters log transformations were made before analysis. The model included random effects for subjects as well as sessions (i.e. flight days) for a given subject. From each fitted model, we made multiple comparisons between the interaction of Braslet (on, off) and test condition (baseline, Valsalva, Mueller) for each outcome parameter. As was the case in Phase I, correction for multiple inferences on 3 comparisons for each of 27 parameters had to be made. To do this, we again used the method of Benjamini, et. al(25), but 
this time with the more conservative FDR control of 5\%. In this case the critical p-value threshold was 0.0075, again with a nominal level of $\alpha=0.05$. Estimated values of Braslet effects (\% change in mean \pm SE) are shown in Table 2 for each parameter and test condition.

Significant effects $(\mathrm{p}<0.0075)$ of test condition as identified by the Benjamini, et al. multiplecomparison procedure are in shown on the right.

\section{Results}

Approximately 7000 measurements were made to obtain $27 \mathrm{ECHO}$ and peripheral vascular parameters acquired during the 15 ultrasound sessions on nine subjects. (See Table 1) Out of the 17 sessions actually scheduled, only 15 were successfully conducted which produced a useful set of data.

Some anomalies include:

- One session - not performed due to ultrasound hardware failure

- One session - cardiac image data were not captured properly due to a procedural error

- One session - no ECG tracing was obtained with vascular images

- One session - last $50 \%$ of data lost due to hardware failure.

- One session - not performed due to scheduling constraints

- One session - respiratory maneuvers not properly synchronized causing difficulties with data analysis.

\section{Phase 1 Analysis: Effect of Braslet alone on Respiratory Maneuvers}

\section{Insert Table 1 here}

After correcting for multiple testing (Statistical Methods), the Braslet cuff was identified as producing a statistically significant effect on the mean response during at least one type of maneuver (including baseline) in 10 of the 27 parameters measured. In particular significant decreases with application of the cuff were observed in cardiac output, LV Stroke volume, left lateral E', Mitral A and E wave velocity, and right IVRT during baseline; left lateral $\mathrm{A}^{\prime}$ and $\mathrm{E}^{\prime}$ during the modified Valsalva maneuver; and left lateral E', right IVRT, and right TEI index during the modified Mueller maneuver. Significant increases were observed in Mitral deceleration time (baseline and Valsalva) and in FEV V area (baseline) (stars in Table 1). 


\section{Phase 2 Analysis: Comparison of Valsalva, Mueller and Baseline} +Maneuvers with and without Braslet

Again after correction for multiple testing, we observed statistical evidence that the effect of the Braslet cuff (on vs. off) was significantly different in 7 parameters between at least two of the maneuvers (including baseline) (last column of Table 1). More specifically, parameters showing evidence of this differential change were cardiac output, heart rate, IJV area, LV diastolic volume, left lateral S', mitral deceleration time, and right lateral E'.

\section{Braslet Release}

After completing all the imaging components with maneuvers in Braslet-OFF and Braslet-ON states, the cardiac probe was positioned to get a continuous four-chamber view. The operator crewmember rapidly released the Velcro straps on both Braslet cuffs, and the following 10 cardiac cycles were recorded. Unfortunately, the four-chamber view $(n=6)$ was difficult to maintain during the Braslet resulting in poor image quality, therefore it was replaced with the more stable Tissue Doppler $(\mathrm{n}=9)$.

\section{Discussion}

Realtime remote expert guidance of the non-physician crewmembers allowed the ultrasound operators to obtain high quality images and data that were critical to the success of this study. Herault et al(26) reported that despite adequate training of Mir cosmonauts before flight, the quality of the ultrasound obtained for in-flight studies prevented them from determining changes in cardiac parameters of less than 10\%. In our study, the use of experts with substantial remote image acquisition experience and an established and tested system of balanced expertise distribution (pre-flight and just-in-time training, supporting tools and materials, and remote guidance) enabled data acquisition with quality acceptable for real-time assessment and thorough retrospective analysis.

Tissue Doppler (TD) was performed for the first time in space during this investigation. TD was taken from the left ventricular (LV) lateral wall, septum, and right ventricular (RV) free wall. TD is an excellent means of assessing changes in cardiac performance, which is of 
particular importance for long-duration space flight.(Figure 4) Furthermore, TD spectra are easier to obtain and less vulnerable to motion and artifact than 2D echocardiographic views. In our subjects, LV Lateral E' decreased by more than $20 \%$ with the application of the Braslet regardless of the maneuver, and LV Lateral A' decreased only when Braslet was applied and a Valsalva maneuver was performed. RV iso-volumic relaxation time decreased by more than $20 \%$ with the Braslet applied and by more than $30 \%$ when a Mueller maneuver was simultaneously performed. Mitral pulsed wave Doppler velocity of the LV inflow is a loaddependant parameter and decreases in response to reduced preload.(27;28) By manipulating preload with Valsalva and Mueller respiratory maneuvers, a reduction in preload can be demonstrated as smaller velocities and extended relaxation slopes. (Figure 5)

Femoral vein (FV) area increased by $89 \%$ with the application of the Braslet. This change was less pronounced (51\%) when a Valsalva maneuver was compared pre/post Braslet, since pre-Braslet Valsalva already increased the FV area. This observation provides evidence that the Braslet, when worn and calibrated correctly, still allows for thoracic maneuvers to have a demonstrable effect on lower-extremity venous filling. The effect of occlusion cuffs on the lower-extremity venous system compresses the superficial veins more than the deep collateral circulation.(29-31) Herault et al(26) reported the IJV area decreased in space flight with Braslet applied, but we could only effect significant changes in IJV area when the Braslet was applied and thoracic maneuvers were performed.

Cardiac output decreased by $19 \%$ and stroke volume decreased by $12 \%$ when the Braslet was worn, and the heart rate compensation in the increasing direction was observed but was not statistically significant. These findings are consistent with Pourcelot and Pottier(18-20) who reported a decrease in stroke volume ( 20\%) and cardiac output ( 20\%) using echocardiography and Braslet during ashort-duration French-Soviet Salyut flight in 1982. On flight day 4, they used pneumatic thigh cuffs at 40 and $60 \mathrm{mmHg}$, which is similar to the constraining stress of the Braslet measured by Hamilton et al (unpublished results) using balloon pressure transducers(32). Diridollou et al(33) reported that the Braslet used in their HDT study provided the equivalent of $30 \mathrm{mmHg}$ "counterpressure" over the area of it application on the upper thigh. Lindgen et al(34) observed a $3 \%$ increase in leg volume with $12^{\circ}$ head down tilt with thigh cuffs inflated to $50 \mathrm{mmHg}$ for 15 minutes, however these subjects would have been consider hypervolemic compared to microgravity. 
Herault et al(26) measured the effects of wearing the Braslet for five hours on six cosmonauts during a 6-month stay on the Mir space station and reported stroke volume and cardiac output reduction of $15 \%$ and $14 \%$ respectively after one month of space flight. It is interesting to note that these changes became minuscule after three months of space flight, and the Braslet actually increased stoke volume and cardiac output after five months of space flight. They also report that femoral vein area increased by $\sim 20 \%$ with the Braslet applied at one and three months, but five months into the flight this increase was only $9 \%$. We did not observe this trend on any subject and it should be noted that the countermeasures used on the ISS are very effective at preventing muscle atrophy of the lower extremities. Muscle loss may have occurred during the Mir missions, altering the fit and, therefore, the efficacy of the Braslet.

The myocardial performance index (Tei index (35)) is calculated using diastolic and systolic time intervals as a combined measure of myocardial performance. It is a simple and reliable indicator of overall ventricular function. In this study, we calculated the Tei index from the time indices of the RV tissue Doppler. The findings for the RV were similar among all subjects. Normal Tei index at $1 \mathrm{G}$ in the RV is $<0.3(36)$, yet our data showed all but one subject consistently above this level. Hemodynamic manipulation with the Braslet-M as a tool for assessing RV physiology in space proved to be a useful non-invasive evaluation of RV function using the on-board ISS ultrasound. This study, combined with data from Hamilton et al(6), provides a useful reference data set for future clinical and research studies in echocardiography and vascular ultrasound in microgravity.

Although Braslet did not induce a profound change in IJV area despite the obvious reduced cardiac preload, thoracic maneuvers seemed to have a profound additive effect when the Braslet was applied. This implies that the IJV is close to the pressure required to maintain its unstressed volume (i.e., to saturate its filling capacity) and that a Mueller maneuver will decrease its cross-sectional area. The Mueller - Valsalva difference is instructive since Valsalva had a small effect on IJV area, implying the vein was maximally distended despite the reduced cardiac preload caused by the Braslet. Nonetheless, the IJV area decreases significantly when a Mueller maneuver is performed.(Figure 6) This finding is consistent with the IJV, which takes very little CVP to distend it maximally, has a low enough CVP to be manipulated with limited thoracic pressures when Braslet is applied. This can be replicated at the bedside on a healthy patient by observing the change in fullness of the jugular venous pulse when raising or lowering the 
patient's neck by only one inch. Therefore, a Mueller maneuver with Braslet applied in microgravity seems to decrease the CVP acutely to less than $2 \mathrm{mmHg}$. This is consistent with the maintenance of RV preload under normal terrestrial conditions where RV transmural pressures are approximately $1.5 \mathrm{mmHg}$ in humans.(37)

Diridollou et al(33) used high frequency ultrasound to demonstrate the increase in forehead skin thickness (2.8\%) and decrease in tibial skin thickness (3.5\%) when subjects are placed in $6^{\circ} \mathrm{HDT}$. When Braslet was applied during 7 days of HDT, the forehead thickness decreased to $0.6 \%$ of baseline compared to the control group which increased to $6.4 \%$. Arbeille et al $(38 ; 39)$ measured IJV distension and facial edema in a head-down tilt (HDT) study. Compared to supine baseline values they found that facial skin thickness increased by 5\% after seven days of HDT but, after wearing the Braslet for eight hours the skin thickness was reduced by $5 \%$. This agrees with the subjective comments by Herault et al(26), which document that all astronauts who wore the Braslet during their study in space claim to have had a "sensation of comfort." Matsnev et al(40) reported that when Braslet was employed on Soyuz-38 the cosmonauts reported a reduction in space adaptation syndrome symptoms (dizziness, congestion and headaches).

Kirsch et al(14) measured CVP after 22 hours of microgravity during the Spacelab 1 mission and found it to be less than the preflight supine levels in two subjects. They repeated this during the Spacelab D1 missions and found that the CVP again fell to levels below preflight supine 20 to 40 minutes after liftoff. This is consistent with the findings by Buckey et al (4), which found that invasive CVP fell to $2.5 \mathrm{~cm} \mathrm{H}_{2} \mathrm{O}$ immediately at the transition to microgravity on the Space Life Sciences 1 mission. Foldager et al(15) reported that CVP decreased to 6.5 $2.0 \mathrm{mmHg}$ after three hours of microgravity exposure during the Spacelab D2 (n=4) and Space Life Sciences $2(\mathrm{n}=2)$ missions.

The monitoring of the IJV using thoracic maneuvers may replace the terrestrial bedside JVP to determine volume status of the microgravity patient. We agree with the hypothesis of Tyberg et al(41) suggesting a mechanism by which blood volume changes might explain the hypotension seen following return to normal gravity after space flight. Upon entering microgravity, lower-extremity peripheral veins significantly reduce in volume and, because of volume redistribution, distend the right atrium even though CVP is decreased. After some time in space, homeostatic mechanisms may cause a decrease in circulating volume and reduce the 
pressure in the central venous compartment to values below what is found on Earth but adequate to provide the necessary preload for the heart. The new set point for central venous pressure may be a combination of the pressure that provides the external constraint to the lower extremity venous system or the pericardial constraint of the heart under long-duration microgravity conditions. The RV end-diastolic pressure, which approximates the CVP, is mostly determined by pericardial constraint even at low filling pressures.(42) Nonetheless, large changes in intrathoracic pressure manifest themselves more profoundly in microgravity when the Braslet is applied, which is similar to their effect on a hypovolemic patient. Clearly, a human in space who is euvolemic by microgravity standards has less hemodynamic volume reserve compared to their terrestrial counterpart and would be more susceptible to hypovolemic shock for the same intravascular volume loss.

During the Braslet release the four-chamber images consistently show an immediate increase in LV stroke volume, which oscillated in magnitude over several beats and stabilized to a value that was close to the pre-Braslet application. The increased stroke volume after the Braslet release lasts for less than 10 beats, which implies that a significant portion of the sequestered volume in the leg must be sequestered in the extra-vascular space.(Figure 7) These results may be consistent with Fujimoto et al(42), who showed that ventricular interaction modulates LV performance even at low filling pressures. They performed lower-body negative pressure (LBNP) (- $30 \mathrm{mmHg}$ ) on humans and observed a reduction in stroke volume of $3 \%$ despite a transient increase in RV preload. Perhaps the oscillatory nature of the response following Braslet release observed in our subjects may be due to ventricular interaction or a baroreflex response. Hinghofer-Szalkay et al(43) demonstrated that $35 \mathrm{mmHg}$ LBNP could be 'counter balanced" with $27.5^{\circ}$ of HDT. Given that the Braslet is calibrated to counterbalance the effect of $30^{\circ} \mathrm{HDT}$, it can be assumed that it may have an effect on the lower extremities similar to $-30 \mathrm{mmHg}$ of LBNP.

The capability of Braslet cuffs to acutely reduce the global cranial venous filling suggests their possible use in other conditions that would benefit from acute fluid redistribution. Similar to the IJV cross-section in this experiment, a saturated parameter could be reset to gain insight into the extent of the role of venous hyperemia in a number of physiological phenomena encountered in space. Braslet could also represent a therapeutic capability, most likely in combination with other measures, to control certain medical hazards in space. For example, the 
chronically dilated venous system of the neck and presumably other cranial venous structures may play a role in the pathophysiology responsible for the observation of some ISS crewmembers who demonstrate cerebrospinal fluid opening pressures greater than $25 \mathrm{~cm}_{2} \mathrm{O}$ when measured several days postflight and, in a few, remaining elevated even months postflight. Postflight fundoscopic examinations of these affected crewmembers revealed optic disc edema, choroidal folds, or cotton wool spots that did not exist before flight. MRI and ultrasound examinations of the eye in these crewmembers also demonstrated optic disc edema, posterior flattening of the globe, or dilated optic nerve sheaths, which is similar to patients with idiopathic intracranial hypertension. The results of this study suggest that the application of Braslet in combination with thoracic maneuvers may play a role in understanding this poorly understood space flight hazard and/or helping control it by theoretically reducing cranial venous and lymphatic insufficiency.

Another example of a possible use of the Braslet device in microgravity would be for the treatment of high-pressure pulmonary edema since all regions of the lung will be susceptible to pulmonary edema at the same left atrial pressure in microgravity. In terrestrial gravity, when patients have basilar crackles secondary to acute high-pressure pulmonary edema, the mid-lung pressure is typically around $20 \mathrm{~cm} \mathrm{H}_{2} \mathrm{O}$ with the bases closer to $30 \mathrm{~cm} \mathrm{H}_{2} \mathrm{O}$. It may stand to reason, in microgravity, that a higher initial pressure of $30 \mathrm{~cm} \mathrm{H}_{2} \mathrm{O}$ needs to be achieved, but then sudden global alveolar flooding will most likely occur. This implies that, in microgravity, the auscultation of crackles anywhere in the thorax in the setting of fluid overload may be a harbinger of impending fulminant pulmonary edema. The Braslet could help control this condition in a manner similar to nitrates until diuretics can be used.

\section{Conclusion}

The Braslet device reduces the effective circulating volume by sequestering fluid in the lower extremities, as directly observed by vascular ultrasound and supported by the reduced preload indices measured by echocardiography. Vascular ultrasound confirmed reduced distention of the jugular venous system and increased sensitivity of the jugular vein area to thoracic maneuvers. All of these findings combined with subjective comments from crewmembers that the Braslet seems to reduce the sense of "fullness in the head," suggests that the relative cranial venous insufficiency caused by microgravity is partially alleviated by the 
Braslet. The hazards secondary to wearing the occlusive Braslet cuffs in microgravity for an extended time (greater than 1 hour) are not known, and will be investigated in future studies.

Following conservative safety considerations, this experiment established an effective set of modifications to the widely used respiratory maneuvers, introducing their open-glottis, limited-pressure modifications. The conservative physiologically gentle and unequivocal respiratory maneuvers were shown to cause a statistically significant hemodynamic modifications.

A statistically significant reduction in jugular venous filling (and probably, pressure"desaturation") also observed during Braslet application, which coupled with the added influence of negative airway pressure to consistently collapse the internal jugular vein. This effect has important diagnostic and therapeutic implications for space medicine, and should be further studied from both perspectives.

Remotely guided ultrasound provides an effective and objective means of measuring the physiological effects of the Braslet and produces data quality that is superior to previous Braslet investigations in space. Valsalva and Mueller maneuvers may be additional maneuvers needed to observe the patient at the microgravity bedside to determine volume status. 


\section{Figure Legends}

Figure 1. Subject wearing Braslet-M that is contoured to a custom fit and worn bilaterally high on the legs (upper Panel). This example shows the Braslets in the released position. In flight, the Braslets are tightened by the Velcro strap to a preflight setting prescribed in Russia. The setting is optimized to alleviate the increased head ward fluid shift and negative physical symptoms of early space flight. Rheographic waveforms (lower panel) from Russian calibration procedure with Braslet-M tightened to the prescribed setting. Notice how the prescribed setting at -30 degrees head-down tilt is similar to the supine waveform.

Figure 2. Astronaut Dr. Michael R. Barratt, Expedition 19 and 20 flight engineer, is shown performing self scanning echocardiography on the ISS. Dr. Barratt is performing tissue Doppler with the Braslet-M device. Notice he has a thin tube in his mouth for performing controlled thoracic maneuvers.

Figure 3. Graphic representation of the standard Braslet protocol. The cardiac session is performed first and last since it is also used for the Braslet release. This reduces the probe changes to only twice during the whole session. ( $2 \mathrm{D}=2$ Dimension, $\mathrm{PW}=$ Pulse Wave, $\mathrm{TD}=$ Tissue Doppler Jug = Jugular Vein, Fem = Femoral Vein )

Figure 4. Tissue Doppler spectral signal with velocities and time intervals labeled for analysis of the right ventricle free wall.

Figure 5. With Braslet ON, Valsalva and Mueller respiratory maneuvers cause a reduction in preload, which can be demonstrated as smaller velocities and extended relaxation slopes in the mitral valve waveform.

Figure 6. Internal Jugular Vein in cross section at Baseline, with Valsalva and Mueller maneuver. With Braslet-ON, the internal jugular veins responded with smaller changes in area with Valsalva and collapse of the vein with Mueller maneuver.

Figure 7. During the Braslet release an immediate increase in LV stroke volume occurs which oscillates over several beats and then stabilizes to a value that is close to the pre-Braslet Basleine. 


\section{References}

\section{Reference List}

(1) Blomqvist CG. Cardiovascular adaptation to weightlessness. Med Sci Sports Exerc 1983;15(5):428-31.

(2) Bungo MW, Charles JB, Johnson PC. Cardiovascular deconditioning during space flight and the use of saline as a countermeasure to orthostatic intolerance. Aviat Space Environ Med 1985 Oct;56(10):985-90.

(3) Thornton WE, Moore TP, Pool SL. Fluid shifts in weightlessness. Aviat Space Environ Med 1987 Sep;58(9 Pt 2):86-90.

(4) Buckey JC, Gaffney FA, Lane LD, Levine BD, Watenpaugh DE, Wright SJ, et al. Central venous pressure in space. J Appl Physiol 1996 Jul;81(1):19-25.

(5) Buckey JC, Goble RL, Blomqvist CG. A new device for continuous ambulatory central venous pressure measurement. Med Instrum 1987 Aug;21(4):238-43.

(6) Hamilton DR, Sargsyan AE, Martin D., Garcia K, Melton S, Dulchavsky SA. On-Orbit Prospective Echocardiography on International Space Station Crew. Echocardiography Journal 2011; In Print.

(7) Olabi AA, Lawless HT, Hunter JB, Levitsky DA, Halpern BP. The effect of microgravity and space flight on the chemical senses. J Food Sci 2002 Mar;67(2):468-78.

(8) Fincke EM, Padalka G, Lee D, van Holsbeeck M, Sargsyan AE, Hamilton DR, et al. Evaluation of Shoulder Integrity in Space: First Report of Musculoskeletal US on the International Space Station. Radiology 2005 Feb;234(2):319-22.

(9) Foale CM, Kaleri AY, Sargsyan AE, Hamilton DR, Melton S, Martin D, et al. Diagnostic instrumentation aboard ISS: just-in-time training for non-physician crewmembers. Aviat Space Environ Med 2005 Jun;76(6):594-8.

(10) Sargsyan AE, Hamilton DR, Jones JA, Melton S, Whitson PA, Kirkpatrick AW, et al. FAST at MACH 20: Clinical Ultrasound Aboard the International Space Station. J Trauma 2005 Jan;58(1):35-9.

(11) Chiao L, Sharipov S, Sargsyan AE, Melton S, Hamilton DR, McFarlin K, et al. Ocular examination for trauma; clinical ultrasound aboard the International Space Station. J Trauma 2005 May;58(5):885-9.

(12) Sargsyan AE, Dulchavsky SA, Jones JA, Hamilton DR, Roden S, Melton S, et al. Abdominal Imaging with Ultrasound on the International Space Station. Aviat Space Environ Med 2006 Mar; 77th Annual Scientific Meetings, ASMA, Orlando FL.

(13) Pasdeloup T, Mas M, Stevenin H. Remote assistance experiment during the manned space flight Altair. Acta Astronaut 1995 Oct;36(8-12):625-8.

(14) Kirsch KA, Rocker L, Gauer OH, Krause R, Leach C, Wicke HJ, et al. Venous pressure in man during weightlessness. Science 1984 Jul 13;225(4658):218-9.

(15) Foldager N, Andersen TA, Jessen FB, Ellegaard P, Stadeager C, Videbaek R, et al. Central venous pressure in humans during microgravity. J Appl Physiol 1996 Jul;81(1):408-12.

(16) Atkov OYu, Bednenko VS, Fomina GA. Ultrasound techniques in space medicine. Aviat Space Environ Med 1987 Sep;58(9 Pt 2):69-73. 
(17) Gazenko OG, Schulzhenko EB, Grigoriev AI, Atkov OYu, Egorov AD. Review of basic medical results of the Salyut-7--Soyuz-T 8-month manned flight. Acta Astronaut 1988 Feb;17(2):155-60.

(18) Pottier JM, Patat F, Arbeille P, Pourcelot L, Massabuau P, Guell A, et al. Cardiovascular system and microgravity simulation and inflight results. Acta Astronaut 1986 Jan;13(1):47-51.

(19) Pourcelot L, Pottier JM, Arbeille P, Patat F, Berson M, Roncin A, et al. [Cardiovascular function in astronauts (Mission STG 51 G--June 1985)]. Bull Acad Natl Med 1986 Mar;170(3-4):341-4.

(20) Pourcelot L, Patat F, Defontaine M, Gregoire JM, Berson M. Microgravity. Ultrasound Med Biol 2000 May;26 Suppl 1:S144-S146.

(21) Arbeille P, Fomina G, Pottier JM, Bystrov V, Patat F, Kokova N, et al. Cardiovascular disturbances induced by a 25 days spaceflight and a one month head down tilt. Physiologist 1991 Feb;34(1 Suppl):151 2.

(22) Kilburn KH, Sieker HO. Hemodynamic effects of continuous positive and negative pressure breathing in normal man. Circ Res 1960 May;8:660-9.

(23) The American Registry for Diagnostic Medical Sonography. http://www.ardms.org . 1-1-2011. 3-29-2011. Ref Type: Online Source

(24) National Board of Echocardiography. 11-1-2010.

Ref Type: Online Source

(25) Benjamini Y, Yekutieli D. The Control of the False Discovery Rate in Multiple Testing Under Dependency. Annals of Statistics 2001;29(4):1165-88.

(26) Herault S, Fomina G, Alferova I, Kotovskaya A, Poliakov V, Arbeille P. Cardiac, arterial and venous adaptation to weightlessness during 6-month MIR spaceflights with and without thigh cuffs (bracelets). Eur J Appl Physiol 2000 Mar;81(5):384-90.

(27) Hurrell DG, Nishimura RA, Ilstrup DM, Appleton CP. Utility of preload alteration in assessment of left ventricular filling pressure by Doppler echocardiography: a simultaneous catheterization and Doppler echocardiographic study. J Am Coll Cardiol 1997 Aug;30(2):459-67.

(28) Nagueh SF. Echocardiographic assessment of left ventricular relaxation and cardiac filling pressures. Curr Heart Fail Rep 2009 Sep;6(3):154-9.

(29) Buckey JC, Peshock RM, Blomqvist CG. Deep venous contribution to hydrostatic blood volume change in the human leg. Am J Cardiol 1988 Sep 1;62(7):449-53.

(30) Gazenko OG, Shumakov VI, Kakurin LI, Katkov VE, Chestukhin VV, Nikolayenko EM, et al. Effects of various countermeasures against the adverse effects of weightlessness on central circulation in the healthy man. Aviat Space Environ Med 1982 Jun;53(6):523-30.

(31) Katkov VE, Chestukhin VV, Nikolaenko EM, Gvozdev SV, Rumiantsev VV. [Effect of negative pressure and occluding cuffs on the intravascular pressure in the lower extremity of the healthy human being]. Kosm Biol Aviakosm Med 1981 Jun;15(4):9-12.

(32) Hamilton DR, deVries G, Tyberg JV. Static and dynamic operating characteristics of a pericardial balloon. J Appl Physiol 2001 Apr;90(4):1481-8. 
(33) Diridollou S, Maillet A, Pavy-Le Traon A, Louisy F, Black D, Berson M, et al. Use of thigh pressure cuffs to modulate simulated microgravity-induced changes in the skin measured with high-resolution B-scan ultrasound. Eur J Ultrasound 2001 Jul;13(3):215-26.

(34) Lindgren KN, Kraft D, Ballard RE, Tucker A, Hargens AR. Venoconstrictive thigh cuffs impede fluid shifts during simulated microgravity. Aviat Space Environ Med 1998 Nov;69(11):1052-8.

(35) Tei C, Nishimura RA, Seward JB, Tajik AJ. Noninvasive Doppler-derived myocardial performance index: correlation with simultaneous measurements of cardiac catheterization measurements. J Am Soc Echocardiogr 1997 Mar;10(2):169-78.

(36) Haddad F, Hunt SA, Rosenthal DN, Murphy DJ. Right ventricular function in cardiovascular disease, part I: Anatomy, physiology, aging, and functional assessment of the right ventricle. Circulation 2008 Mar 18;117(11):1436-48.

(37) Hamilton DR, Dani RS, Semlacher RA, Smith ER, Kieser TM, Tyberg JV. Right atrial and right ventricular transmural pressures in dogs and humans. Effects of the pericardium. Circulation 1994 Nov;90(5):2492500 .

(38) Arbeille P, Diridillou S, Herault S, Fomina G, Roumy J, Alferova I. Effect of the thigh-cuffs on the carotid artery diameter jugular vein section and facial skin edema: HDT study. J Gravit Physiol 1999 Jul;6(1):3940.

(39) Arbeille P, Herault S, Fomina G, Roumy J, Alferova I, Gharib C. Influences of thigh cuffs on the cardiovascular system during 7-day head-down bed rest. J Appl Physiol 1999 Dec;87(6):2168-76.

(40) Matsnev EI, Yakovleva IY, Tarasov IK, Alekseev VN, Kornilova LN, Mateev AD, et al. Space motion sickness: phenomenology, countermeasures, and mechanisms. Aviat Space Environ Med 1983 Apr;54(4):312-7.

(41) Tyberg JV, Hamilton DR. Orthostatic hypotension and the role of changes in venous capacitance. Med Sci Sports Exerc 1996 Oct;28(10 Suppl):S29-S31.

(42) Fujimoto N, Shibata S, Hastings JL, Carrick-Ranson GC, Bhella PS, Palmer MD, et al. Effects of Pericardial Constraint and Ventricular Interaction on Left Ventricular Hemodynamics in the Unloaded Heart. Am J Physiol Heart Circ Physiol 2011 Mar 11.

(43) Hinghofer-Szalkay H, Haditsch B, Loder I, Pilz K, Rossler A, Jezova D. Head down tilt at -6 degrees to -24 degrees can neutralize the cardiovascular effects of LBNP at -15 or $-35 \mathrm{mmHg}$. Aviat Space Environ Med 2004 Nov;75(11):947-51. 
Figures
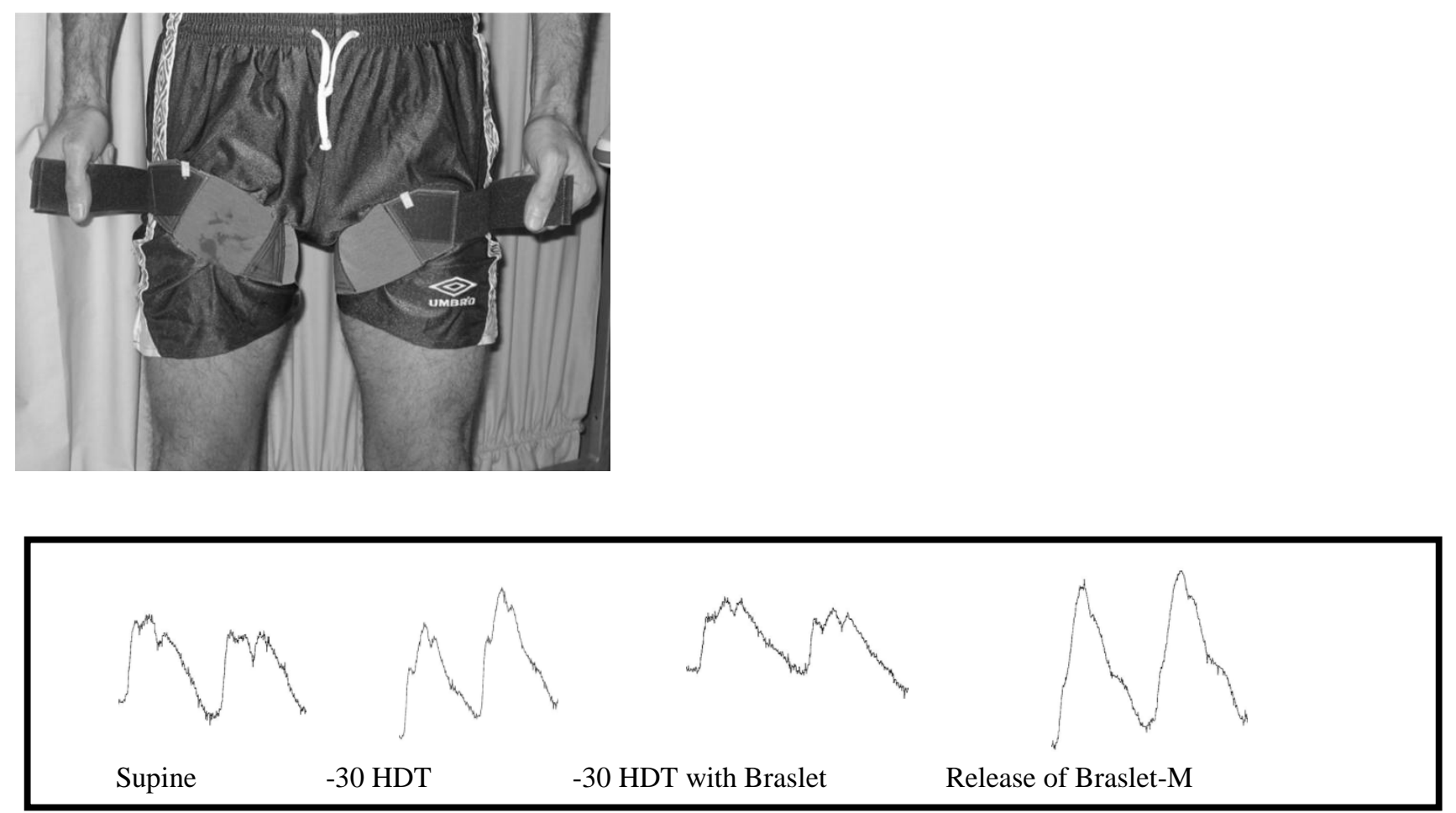

Figure 1. 


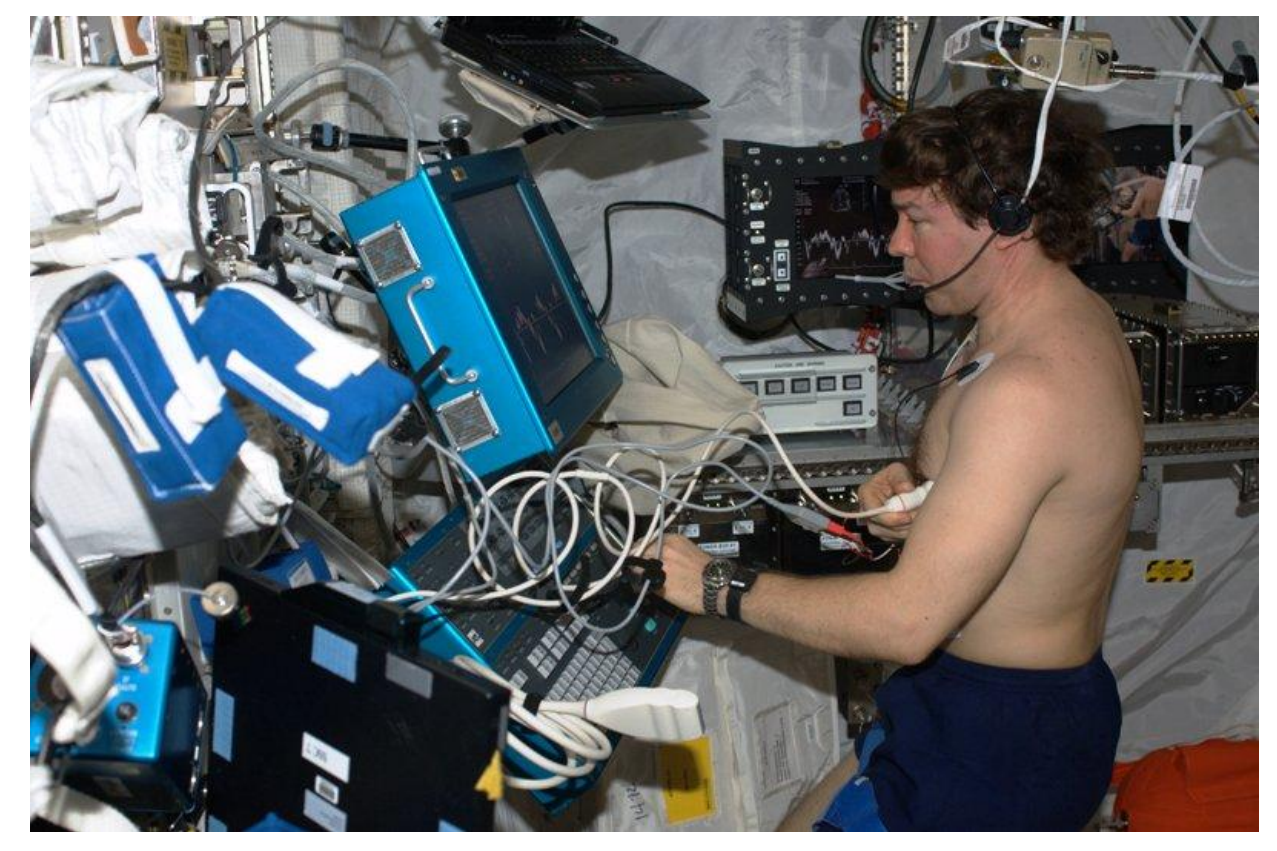

Figure 2 


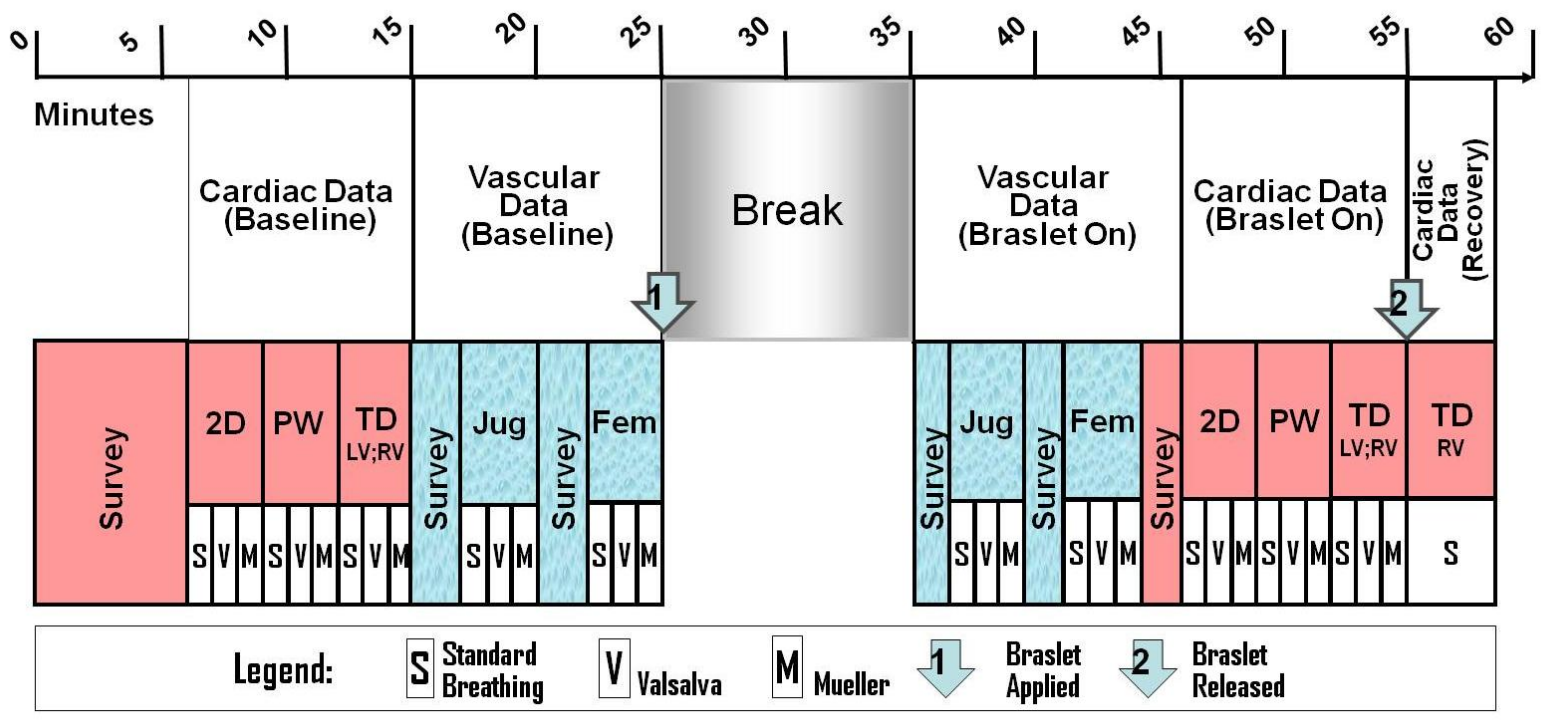

Figure 3 


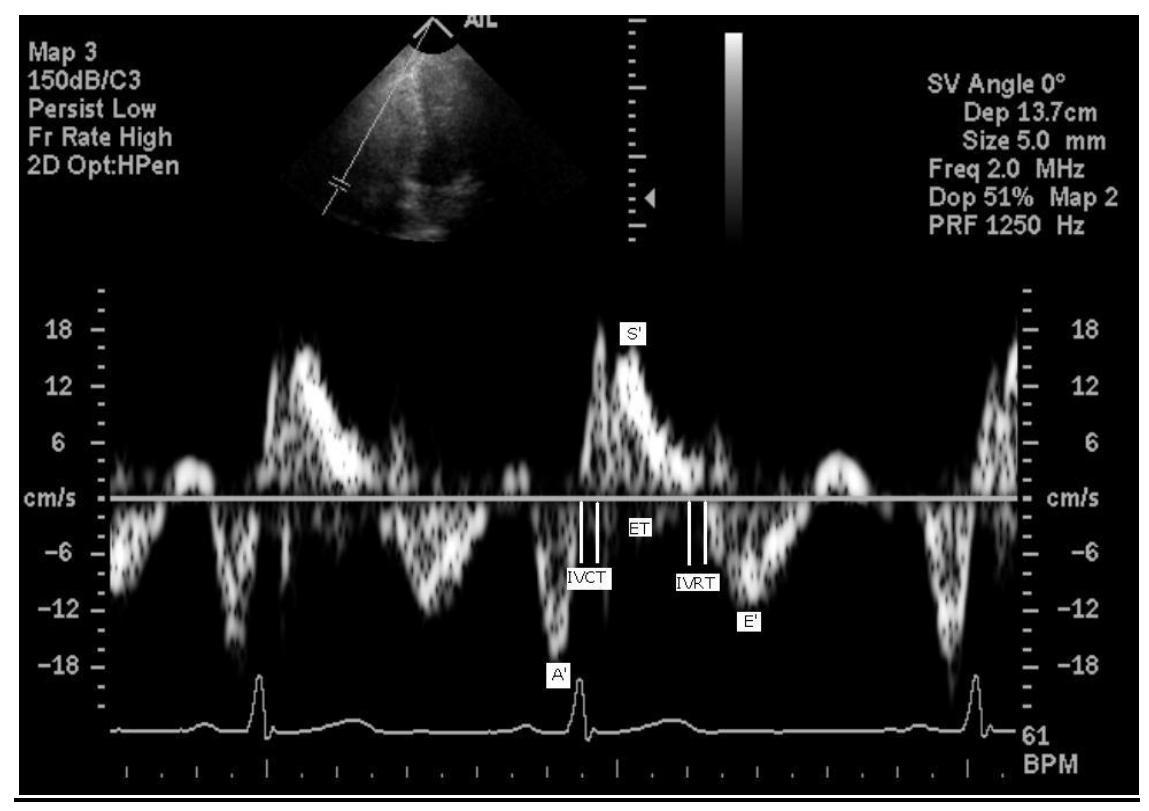

Figure 4 

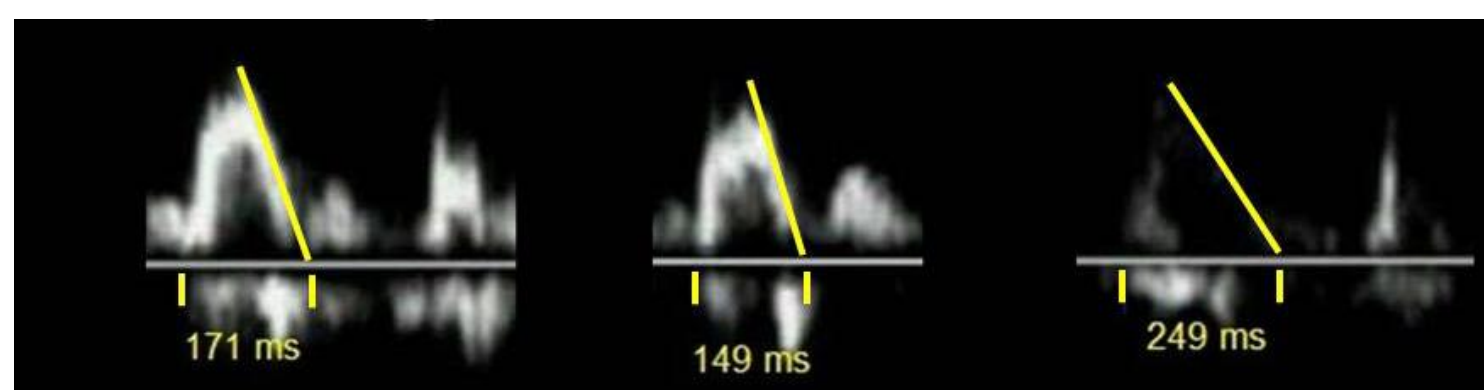

No Maneuver

Valsalva Maneuver

Muller Maneuver
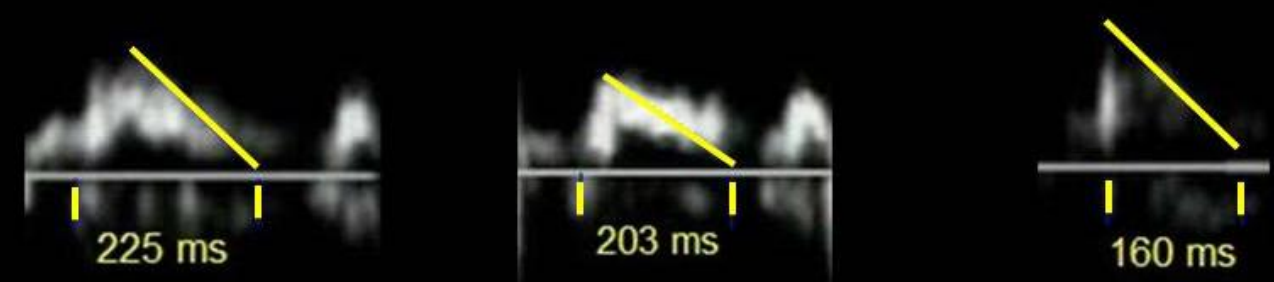

Figure 5. 


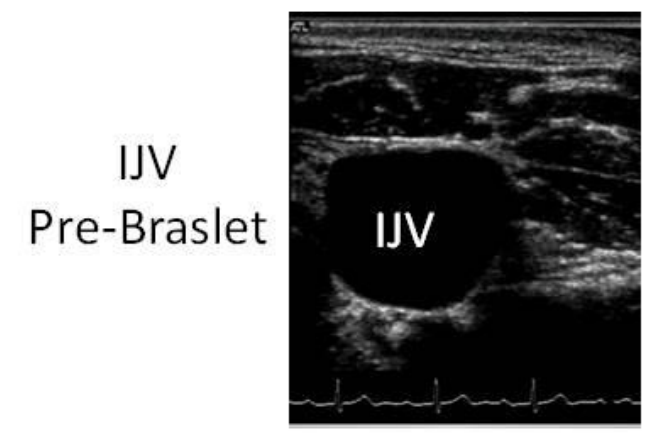

Baseline

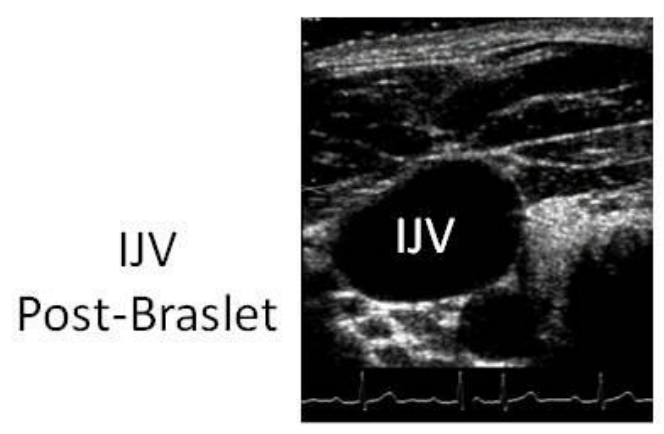

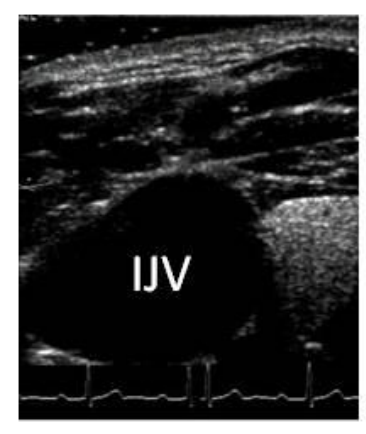

Valsalva

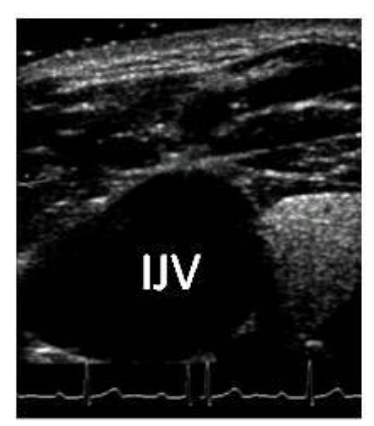

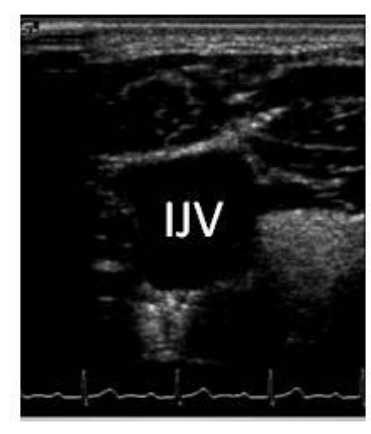

Mueller

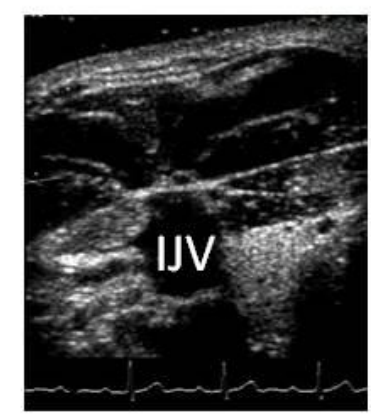

Figure 6 


\section{Stroke Volume with Braslet Release}

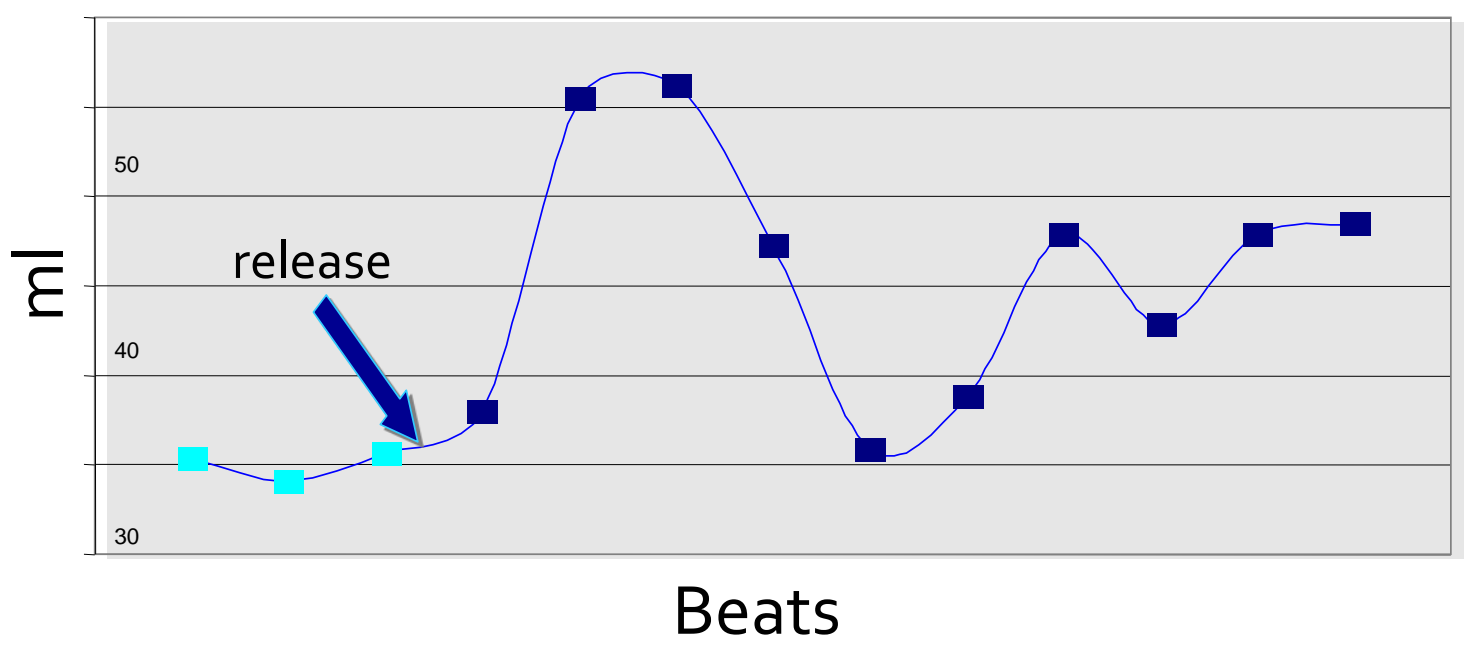

Figure 7. 


\begin{tabular}{|c|c|c|c|c|c|c|c|}
\hline \multirow{2}{*}{ outcome measure } & \multicolumn{2}{|c|}{ baseline } & \multicolumn{2}{|c|}{ Valsalva } & \multicolumn{2}{|c|}{ Mueller } & \multirow{2}{*}{$\begin{array}{c}\text { Difference } \\
\text { Test }\end{array}$} \\
\hline & $\%$ change & (se) & \% change & (se) & $\%$ change & (se) & \\
\hline Cardiac Output & $\star-20.0$ & 4.0 & 0.1 & 5.1 & -4.3 & 5.2 & $\mathrm{VB}, \mathrm{MB}$ \\
\hline FEM V Area & $\star 64.1$ & 11.2 & $\star 47.4$ & 9.3 & 40.9 & 8.7 & \\
\hline Heart Rate & -3.3 & 0.8 & 1.3 & 0.8 & -0.9 & 0.8 & VB \\
\hline IJV Area & -23.4 & 5.8 & -6.2 & 5.1 & -48.3 & 11.4 & $\mathrm{MB}, \mathrm{MV}$ \\
\hline LV Diastolic Volume & -7.7 & 2.7 & 4.0 & 3.5 & -1.8 & 3.3 & VB \\
\hline LV Ejection Fraction & -6.7 & 2.5 & -4.4 & 2.9 & -1.1 & 3.0 & \\
\hline LV Stroke Volume & $\star-12.7$ & 3.7 & 0.1 & 4.9 & -4.8 & 4.7 & \\
\hline LV Systolic Volume & 7.1 & 5.2 & 12.0 & 6.7 & 5.2 & 6.0 & \\
\hline$\%$ change in LV area & -11.0 & 2.9 & -5.2 & 3.6 & -4.3 & 3.4 & \\
\hline Left ET & -3.6 & 1.4 & -5.7 & 1.4 & -2.1 & 1.5 & \\
\hline Left IVCT & 1.7 & 3.3 & -2.5 & 3.4 & 2.3 & 3.6 & \\
\hline Left IVRT & 11.1 & 4.8 & 2.1 & 4.2 & 12.1 & 5.2 & \\
\hline Left Lateral A' & -10.0 & 2.5 & $\star-12.8$ & 2.6 & -4.8 & 2.7 & \\
\hline Left Lateral E' & $\star-27.4$ & 2.7 & $\star-28.3$ & 3.1 & $\star-21.3$ & 2.9 & \\
\hline Left Lateral S' & -1.5 & 3.0 & 11.3 & 3.4 & -5.9 & 2.9 & $\mathrm{VB}, \mathrm{MV}$ \\
\hline Left Tei Index & 11.4 & 4.3 & 9.3 & 4.2 & 9.1 & 4.5 & \\
\hline Mitral A Wave Velocity & $\star \quad-9.5$ & 2.9 & -11.0 & 3.0 & -6.8 & 3.0 & \\
\hline Mitral Decel Time & $\star 11.4$ & 3.1 & -0.6 & 2.5 & -2.7 & 2.9 & $\mathrm{VB}, \mathrm{MB}$ \\
\hline Mitral E Wave Velocity & $\star-16.0$ & 2.5 & -14.2 & 2.9 & -12.0 & 2.5 & \\
\hline \% change in RV area & 2.1 & 5.7 & 12.9 & 7.3 & -0.1 & 6.6 & \\
\hline Right ET & -0.9 & 2.3 & -1.7 & 2.3 & 0.9 & 2.5 & \\
\hline Right IVCT & -20.1 & 4.1 & -10.6 & 4.7 & -7.6 & 5.2 & \\
\hline Right IVRT & $\star-22.0$ & 6.8 & -22.5 & 7.1 & $\star-34.4$ & 7.8 & \\
\hline Right Lateral A' & -0.2 & 3.9 & -8.7 & 3.8 & -2.4 & 4.1 & \\
\hline Right Lateral E' & 2.1 & 3.3 & -8.9 & 3.2 & -1.9 & 3.3 & VB \\
\hline Right Lateral S' & 1.9 & 4.2 & 14.9 & 5.1 & 2.0 & 4.5 & \\
\hline Right Tei index & -19.8 & 4.0 & -11.5 & 4.4 & $\star-20.5$ & 4.8 & \\
\hline
\end{tabular}

VB: Valsalva effect of Braslet significantly different from baseline effect of Braslet $(p<.0075)$. MB: Mueller effect of Braslet significantly different from baseline effect of Braslet $(p<.0075)$. $M V$ : Mueller effect of Braslet significantly different from Valsalva effect of Braslet $(p<.0075)$. $\star$ = considered a significant change due to the BRASLET OFF/ON treatment

Table 1 